\title{
Bioinformatics analysis of Brucella vaccines and vaccine targets using VIOLIN
}

\author{
Yongqun $\mathrm{He}^{*}$, Zuoshuang Xiang \\ From Asia Pacific Bioinformatics Network (APBioNet) Ninth International Conference on Bioinformatics \\ (InCoB2010) \\ Tokyo, Japan. 26-28 September 2010
}

\begin{abstract}
Background: Brucella spp. are Gram-negative, facultative intracellular bacteria that cause brucellosis, one of the commonest zoonotic diseases found worldwide in humans and a variety of animal species. While several animal vaccines are available, there is no effective and safe vaccine for prevention of brucellosis in humans. VIOLIN (http:// www.violinet.org) is a web-based vaccine database and analysis system that curates, stores, and analyzes published data of commercialized vaccines, and vaccines in clinical trials or in research. VIOLIN contains information for 454 vaccines or vaccine candidates for 73 pathogens. VIOLIN also contains many bioinformatics tools for vaccine data analysis, data integration, and vaccine target prediction. To demonstrate the applicability of VIOLIN for vaccine research, VIOLIN was used for bioinformatics analysis of existing Brucella vaccines and prediction of new Brucella vaccine targets.
\end{abstract}

Results: VIOLIN contains many literature mining programs (e.g., Vaxmesh) that provide in-depth analysis of Brucella vaccine literature. As a result of manual literature curation, VIOLIN contains information for 38 Brucella vaccines or vaccine candidates, 14 protective Brucella antigens, and 68 host response studies to Brucella vaccines from 97 peerreviewed articles. These Brucella vaccines are classified in the Vaccine Ontology (VO) system and used for different ontological applications. The web-based VIOLIN vaccine target prediction program Vaxign was used to predict new Brucella vaccine targets. Vaxign identified 14 outer membrane proteins that are conserved in six virulent strains from B. abortus, B. melitensis, and B. suis that are pathogenic in humans. Of the 14 membrane proteins, two proteins (Omp2b and Omp31-1) are not present in B. ovis, a Brucella species that is not pathogenic in humans. Brucella vaccine data stored in VIOLIN were compared and analyzed using the VIOLIN query system.

Conclusions: Bioinformatics curation and ontological representation of Brucella vaccines promotes classification and analysis of existing Brucella vaccines and vaccine candidates. Computational prediction of Brucella vaccine targets provides more candidates for rational vaccine development. The use of VIOLIN provides a general approach that can be applied for analyses of vaccines against other pathogens and infection diseases.

\section{Background}

Brucella is a Gram-negative, facultative intracellular bacterium that causes brucellosis in humans and animals [1]. Brucella are taxonomically placed in the alpha-2 subdivision of the class Proteobacteria. Traditionally there are six species of Brucella based on the

\footnotetext{
* Correspondence: yongqunh@umich.edu

Unit for Laboratory Animal Medicine, Department of Microbiology and Immunology, and Center for Computational Medicine and Bioinformatics, University of Michigan Medical School, Ann Arbor, MI 48109, USA
}

preferential host specificity: B. melitensis (goats), B. abortus (cattle), B. suis (swine), B. canis (dogs), B. ovis (sheep) and B. neotomae (desert mice). The first four species listed in decreasing order of severity are pathogenic to humans making brucellosis a zoonotic disease. These bacteria are also amenable for use in biological warfare and bio-terrorism. Recently, two new species B. cetaceae (cetacean) and B. pinnipediae (seal) have been described [2]. Complete genome sequences of 10 Brucella strains are currently available in the NCBI 
RefSeq database. Four genomes from B. abortus, $B$. melitensis, and $B$. suis have been extensively analyzed [3-6]. While animal brucellosis vaccines are commercially available, there is no effective and safe human vaccine against virulent Brucella infections. Extensive studies on Brucella have recently been concentrated on understanding the mechanisms for protective Brucella immunity and the development of effective human brucellosis vaccines.

VIOLIN (http://www.violinet.org) is a web-based vaccine database and analysis system. VIOLIN contains general information on microbial pathogenesis, host ranges, and host protective immunity, as well as vaccine-specific information such as vaccine type, preparation method, genetically engineered genes, and host responses in various animal models. VIOLIN contains information about 454 vaccines and vaccine candidates for 73 pathogens. VIOLIN contains many bioinformatics tools for vaccine literature mining, vaccine data analysis and integration, and vaccine target prediction. For example, VIOLIN includes Vaxmesh and Vaxpresso programs that may be used to mine vaccine literature based on $\mathrm{MeSH}$ controlled vocabulary and natural language processing (NLP), respectively. Dr. Yongqun He, the founder of the VIOLIN initiated and leads communitybased development of the Vaccine Ontology to support vaccine integration and automated reasoning. A webbased vaccine target prediction program Vaxign available in VIOLIN is used to predict vaccine targets based on genome sequence analysis using a reverse vaccinology strategy.

As of May 13, 2010, more than 2,000 Brucella vaccine-related literature papers were searchable in PubMed, and 10 Brucella genomes have been published in the NCBI RefSeq database. To support Brucella vaccine research and development, we systematically curated from the literature existing Brucella vaccine information, which are stored in VIOLIN for query and further analyses. Different VIOLIN tools are also used to analyze Brucella vaccines and predict new vaccine targets.

\section{Results}

\section{Brucella vaccine literature mining in VIOLIN}

All Brucella vaccine-related articles were downloaded from PubMed and stored in VIOLIN. Information for these articles was processed and used for varying literature mining applications in VIOLIN. For example, Vaxmesh, a MeSH-based vaccine literature visualization and mining tool in VIOLIN, was used (Figure 1). The Medical Subject Headings (MeSH; http://www.nlm.nih.gov/ mesh/) is the controlled vocabulary thesaurus developed by the National Library of Medicine (NLM) to index articles deposited for the MEDLINE/PubMed database.
There are over 25,000 MeSH terms organized in a hierarchical fashion based on 15 top-level categories. The $\mathrm{MeSH}$ hierarchical structure permits literature searching at various levels of specificity. Vaxmesh provides an interactive web interface for users to locate articles using $\mathrm{MeSH}$ terms in a hierarchical MeSH tree structure. Figure 1 demonstrates a MeSH hierarchy for the term "Gene Deletion". This major MeSH term is associated with five papers in Brucella vaccine area (Figure 1A-1B). A click on the MeSH term links the program to another VIOLIN web page that reveals detailed information about each of the five papers. A web link to PubMed is also available (Figure 1C). According the $\mathrm{MeSH}$ indexing, those articles associated with Brucella vaccines also cover different areas such as anatomy (261 articles), physical sciences (194 articles), and geographic locations (47 articles) (Figure 1).

Vaxperts is a new MeSH-based VIOLIN program that provides a literature-based social network of vaccine experts based on their publication records in PubMed. Vaxperts allows vaccine experts to find their co-authors and co-authors's co-authors of shared publications. This approach facilitates collaborative vaccine research and development. For example, a search for the keyword "Brucella" in Vaxperts resulted in the listing of 2454 authors that have contributed to at least one Brucella vaccine article.

VIOLIN also contains three additional literature mining programs. These are: Vaxpresso, a natural language processing (NLP)-based vaccine literature mining program; VIOLIN Litesearch, an advanced keywordand category-based search for vaccine literature; and Vaxlert, a literature alert program that provides periodical literature updates through Emails based on the specification of a VIOLIN user.

\section{Brucella vaccines curated in VIOLIN}

With many literature mining programs available in VIOLIN, it is possible to make manual curation of Brucella vaccine information more efficient. Brucella vaccine curation was performed using a web-based literature mining and curation system called Limix $[7,8]$. Limix was developed to efficiently combine semi-automatic literature mining, manual curation, and data submission. . All curated data includes references. The curated data is published in VIOLIN and available for query only after it is critically reviewed and verified by an expert.

VIOLIN contains 38 curated Brucella vaccines or vaccine candidates that have been officially licensed or proven to provide protection in an animal model (Table 1). Specifically, VIOLIN includes $20 \mathrm{~B}$. abortus vaccines, 16 $B$. melitensis vaccines, and two $B$. suis vaccines. Among them, four Brucella vaccines are licensed for commercial uses in cattle, sheep, goat, and pigs. All others are 


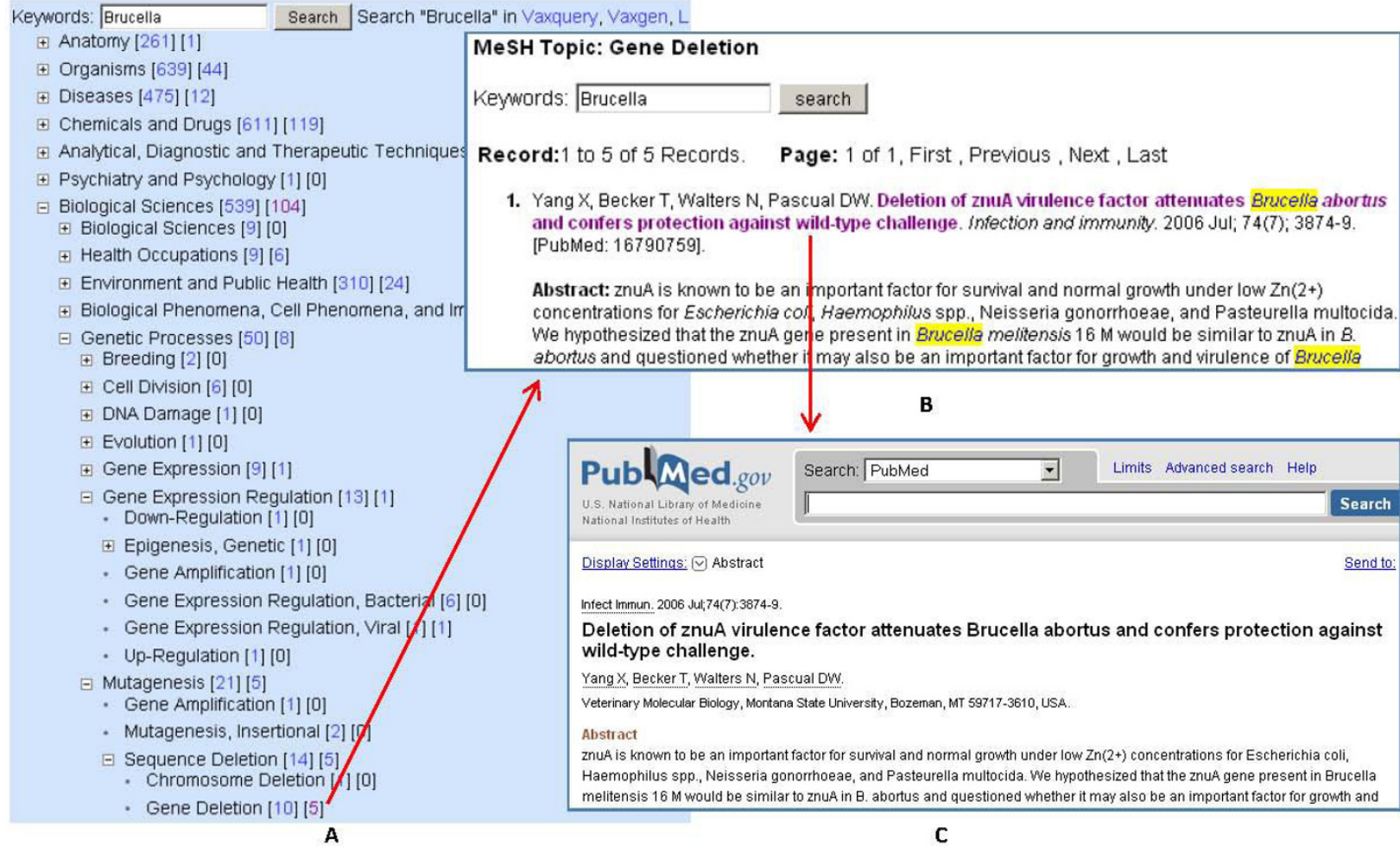

1. Yang $X$, Becker $T$, Walters $N$, Pascual DW Deletion of znuA virulence factor attenuates Brucella abortus and confers protection against wild-type challenge. Infection and immunity. 2006 Jul; 74(7); 3874-9. [PubMed: 16790759].

Abstract: znuA is known to be an important factor for sunvival and normal growth under low $\mathrm{Zn}(2+)$ concentrations for Escherichia col Haemophilus spp. Neisseria gonorrhoeae, and Pasteurella multocida We hypothesized that the znuA ger e present in Brucella meitensis $16 \mathrm{M}$ would be similar to znuA in $B$ abortus and questioned whether i may also be an important factor for growth and virulence of Brucella

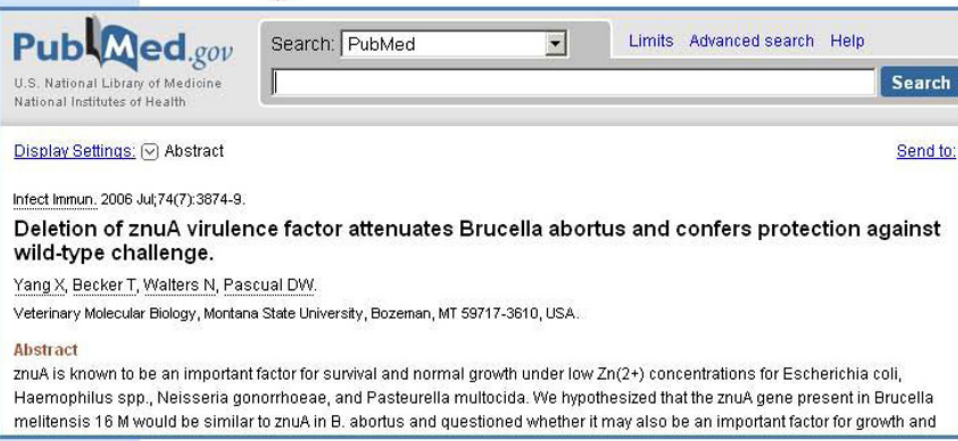

Figure 1 Vaxmesh analysis of Brucella vaccine papers associated with the MeSH term "Gene Deletion". (A) Visualization of MeSH hierarchy in Vaxmesh after keyword "Brucella" search; (B) The two clickable numbers next to each MeSH term links to all publications with the term as a MeSH term or a major MeSH term, respectively. A click on "5" next to the MeSH term "Gene Deletion" links to another page with detailed citation information; (C) The PubMed record is accessible after a click on an article title in (B).

research vaccines which have been demonstrated to induce protection in vivo against virulent Brucella challenges at least in some laboratory models (mostly in the mouse model). In terms of vaccine types, $1,8,10$, and 19 vaccines are bacterial vector vaccine, DNA vaccines, subunit vaccines, and live attenuated vaccines, respectively.

\section{Ontology representation of Brucella vaccines}

A biomedical ontology represents the consensus-based controlled vocabularies of terms and relations which are logically formulated in such a way as to promote automated reasoning. Ontologies are able to structure complex biomedical domains and relate the myriad of data to shared understanding of biomedicine. Ontologies can be used for different purposes. The Gene Ontology (GO) is a well-known example of an ontology created for the primary purpose of providing controlled and standardized terms for naming different types of biological processes, cellular components, and molecular functions [9]. This ontology allows the common representation of attributes of gene products regardless of species of origin. Creating such ontology-based annotations is highly valuable both for querying databases and analyzing high throughput data. This has a significant impact since as of August 2010, over 2,500 peerreviewed publications are identified through a PubMed search of "Gene Ontology", and approximately 35,000 hits are identified through a Google Scholar search using the same keywords. Ontologies can also be used for representation of encyclopedic knowledge, data exchange, and computational data analysis and reasoning.

The Vaccine Ontology (VO; http://www.violinet.org/ vaccineontology) is a collaborative, community-based ontology in the vaccine domain. VO can be used for vaccine data standardization, integration, and computerassisted reasoning. VO utilizes the Basic Formal Ontology (BFO) (http://www.ifomis.org/bfo), a domainindependent ontology, as an upper level ontology. The VO was developed using the W3C standard Web Ontology Language (OWL) (http://www.w3.org/TR/owl-guide/ ). The latest version of $\mathrm{VO}$ is always available at http:// purl.obolibrary.org/obo/vo.owl. In addition, VO has been listed in the OBO (Open Biomedical Ontologies) website (http://www.obofoundry.org/cgi-bin/detail.cgi? $\mathrm{id}=$ vaccine), and deposited in the NCBO BioPortal (http://bioportal.bioontology.org/virtual/1172). To 
Table 1 Brucella vaccines curated in VIOLIN and listed in VO

\begin{tabular}{|c|c|c|c|c|}
\hline$\#$ & Vaccine names & VO ID & Type & Licensed \\
\hline \multicolumn{5}{|c|}{ Brucella abortus vaccines } \\
\hline 1 & B. abortus DNA vaccine pcDNA-SOD & VO_0000018 & DNA & Research \\
\hline 2 & B. abortus RB51 & VO_0000021 & LA & Licensed \\
\hline 3 & B. abortus strain 19 & VO_0000022 & LA & Licensed \\
\hline 4 & B. abortus DNA vaccine encoding BCSP31, SOD and L7/L12 & VO_0000321 & DNA & Research \\
\hline 5 & B. abortus subunit vaccine using $L 7 / L 12$ & VO_0000323 & Sub & Research \\
\hline 6 & Brucellaabortus bacA mutant & VO_0000347 & LA & Research \\
\hline 7 & B. recombinant SurA protein vaccine & VO_0000358 & Sub & Research \\
\hline 8 & B. recombinant DnaK protein vaccine & VO_0000373 & Sub & Research \\
\hline 9 & B. abortus DNA vaccine using $L 7 / L 12$ and Omp16 & VO_0000374 & DNA & Research \\
\hline 10 & B. abortus DNA vaccine encoding $L 7 / L 12$ and P39 & VO_0000385 & DNA & Research \\
\hline 11 & B. abortus with znuA deletion & VO_0000386 & LA & Research \\
\hline 12 & B. abortus porin-S-LPS & VO_0000403 & Sub & Research \\
\hline 13 & B. abortus RB51WboA & VO_0000404 & LA & Research \\
\hline 14 & Recombinant O. anthropi 49237SOD & VO_0000407 & BT & Research \\
\hline 15 & B. abortus pcDNA-BLS & VO_0000421 & DNA & Research \\
\hline 16 & Escheriosome delivery of B. abortus $L 7 / L 12$ & VO_0000423 & Sub & Research \\
\hline 17 & NPAP Brucella vaccine & VO_0000450 & IA & Research \\
\hline 18 & B. abortus strain RB51SOD & VO_0000720 & LA & Research \\
\hline 19 & B. abortus strain $45 / 20$ & VO_0000723 & LA & Research \\
\hline 20 & B. abortus S19 with P39 deletion & VO_0000826 & LA & Research \\
\hline \multicolumn{5}{|c|}{ Brucella melitensis vaccines } \\
\hline 21 & B. melitensis Rev. 1 with bp26 and omp31 deletions & VO_0001171 & LA & Research \\
\hline 22 & B. melitensis strain VTRM1 & VO_0000300 & LA & Research \\
\hline 23 & B. melitensis lipopolysaccharide vaccine & VO_0000311 & Sub & Research \\
\hline 24 & B. melitensis LPS-GBOMP noncovalent complex & VO_0000312 & Sub & Research \\
\hline 25 & B. melitensis DNA vaccine encoding Omp31 & VO_0000325 & DNA & Research \\
\hline 26 & B. melitensis bp26 deletion vaccine & VO_0000338 & LA & Research \\
\hline 27 & B. melitensis WR201 & VO_0000345 & LA & Research \\
\hline 28 & B. ovis microparticle subunit vaccine & VO_0000354 & Sub & Research \\
\hline 29 & microencapsulated B. melitensis mutant vaccine & VO_0000398 & LA & Research \\
\hline 30 & B. melitensis Bp26 and Tf vaccine & VO_0000411 & Sub & Research \\
\hline 31 & B. melitensis P39 recombinant protein vaccine & VO_0000412 & LA & Research \\
\hline 32 & recombinant chimera BLSOmp31 & VO_0000413 & Sub & Research \\
\hline 33 & B. melitensis DNA vaccine encoding Omp31 boosted with Omp31 & VO_0000436 & DNA & Research \\
\hline 34 & B. melitensis Rev. 1 with P39 deletion & VO_0000633 & LA & Research \\
\hline 35 & B. melitensis strain Rev. 1 & VO_0000710 & LA & Licensed \\
\hline 36 & Brucella DNA vaccine encoding chimera BLSOmp31 & VO_0001144 & DNA & Research \\
\hline \multicolumn{5}{|c|}{ Brucella suis vaccines } \\
\hline 37 & B. suis strain VTRS1 & VO_0000303 & LA & Research \\
\hline 38 & B. suis strain 2 & VO_0000722 & LA & Licensed \\
\hline
\end{tabular}

Note: The abbreviations LA, Sub, DNA, Con, IV, and BV represent live attenuated vaccine, subunit vaccine, DNA vaccine, conjugation vaccine, inactivated vaccine, and bacterial vector vaccine, respectively.

provide a means for users to visualize the definitions and usages of $\mathrm{VO}$ terms and their relations, a VO Browser (http://www.violinet.org/vaccineontology/vobrowser/) was developed.

As with other vaccines, Brucella vaccines in $\mathrm{VO}$ are asserted using single inheritance based on Brucella species. Figure 2A demonstrates the asserted hierarchy of
B. abortus vaccines in VO. As an OWL document, $\mathrm{VO}$ also supports computational inference with an OWL reasoner, such as FACT++ [10]. For example, RB51 is asserted under Brucella abortus vaccine (Figure 2A). Since RB51 has the qualities of 'live' and 'attenuated', it is also inferred as a 'live attenuated Brucella vaccine' using $\mathrm{FACT}++$ (Figure 2B). Figure 2 provides a 


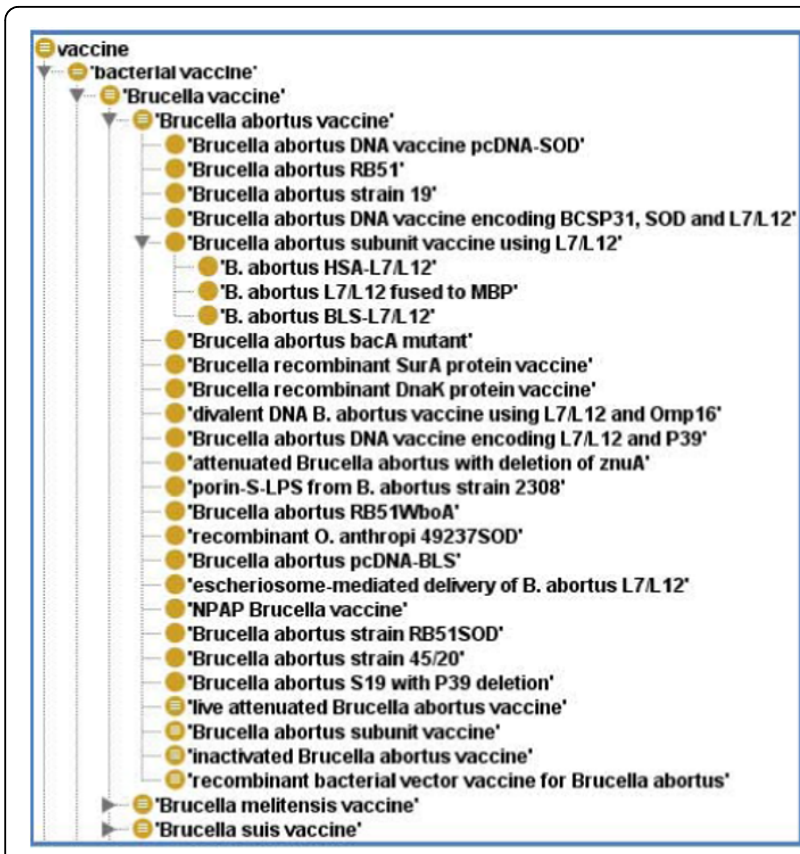

(A)

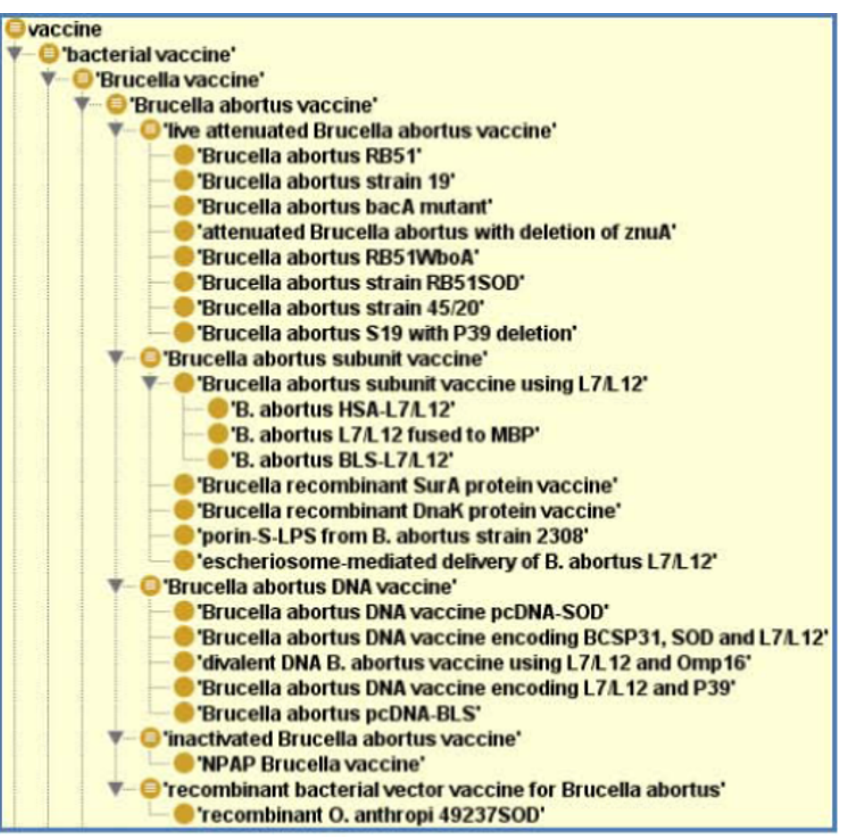

(B)

Figure 2 vo hierarchy of Brucella vaccines. (A) Asserted hierarchy; (B) Inferred hierarchy.

screenshot of Brucella vaccines listed in VO based on computational reasoning.

VO has been used in many applications associated with Brucella vaccines. It can be used to improve PubMed searching efficiency in the vaccine domain. A user case study would be to search "live attenuated Brucella vaccine" in PubMed. As of April 10, 2009, a direct PubMed search of this string of keywords returned 56 papers (or PubMed hits). VO includes 13 live attenuated Brucella vaccines that have the qualities of 'live' and 'attenuated'. When these specific Brucella vaccine terms were also included in a PubMed search, the number of positive paper hits in PubMed increased by more than 10-fold [11]. The combination of VO with SciMiner, a literature mining program, significantly improves PubMed searching efficiency in the general vaccine domain [12]. It was also found that the application of VO dramatically increased the performance of vaccineinduced IFN- interaction networks [13].

Besides vaccine hierarchy, VO can also be used to represent (or model) vaccine investigation. As demonstrated in our two recent reports, vaccine protection investigation can be represented in VO by three continuous steps: vaccination, pathogen challenge, and vaccine efficacy measurement $[14,15]$. A measurement of vaccine efficacy can be assessed by host survival for the pathogens (e.g., Influenza virus) which kill the infected host (e.g., mouse) [14] or by pathogen colony forming units (CFU), a measurement for those pathogens (e.g.,
Brucella) which cannot kill infected host but exhibit diminished replication in a vaccinated host than that in unvaccinated host [15]. It is hypothesized that some parameters will play more important roles than others in determining the protection efficacy of Brucella vaccines. To test this hypothesis, the data for 151 groups of Brucella vaccine protection investigations were collected in VIOLIN from peer-reviewed literature publications and analyzed using ANOVA. Out of 16 parameters, 10 were found statistically significant $(\mathrm{P}$-value $<0.05)$ in contributing to protection based on a statistical ANOVA analysis. Examples of these parameters included vaccine strain, vaccine viability, vaccination route, vaccination dose. However, other six parameters, including IL-12 vaccine adjuvant, mouse sex, vaccination route, animal age, vaccination-challenge interval, and challenge dose, were not found statistically significant (P-value $>0.05)$. A careful study of this use case led to building and validating an ontology-based semantic framework to formally represent ANOVA [15]. Such an ontology-based representation of biomedical data for statistical analysis allows data consistency checking and data sharing in the Semantic Web [16].

\section{Literature curation of Brucella protective antigens}

The VIOLIN Protegen program stores protective antigens that have been verified experimentally to induce protective immunity. Protegen contains 14 protective Brucella antigens (Table 2). Among the 14 Brucella 
Table 2 Brucella protective antigens verified experimentally

\begin{tabular}{llllll}
\hline$\#$ & Symbol & Locus tag & Protein Description & Location & References (PMIDs) \\
\hline 1 & BLS & CAA86936 & Brucella lumazine synthase & Cytoplasm & 11953389 \\
2 & L7/L12 & BRURPL712X & Ribosomal protein L7/L12 & Cytoplasm & 8873388 \\
3 & P39 & ABM67295 & sugar-binding 39-kDa protein & Periplasm & 11447155 \\
4 & Bfr & BAB2_0675 & Ferritin:Bacterioferritin & Cytoplasm & 11447155 \\
5 & Bp26 & BMEI0536 & Periplasmic immunogenic protein & Periplasm & 17239499 \\
6 & DnaK & BruAb1_2100 & Molecular chaperone DnaK & Cytoplasm & 17686554 \\
7 & lalB & BMEl1584 & Invasion protein B & Cytoplasmic membrane & 17049676 \\
8 & Omp16 & BAB1_1707 & Outer membrane protein MotY & Outer membrane & 18981242 \\
9 & Omp19 & BAB1_1930 & Lipoprotein Omp19 & Outer membrane & 18981242 \\
10 & Omp25 & BMEl1249 & 25 kDa outer-membrane immunogenic protein precursor & Outer membrane & 18981242 \\
11 & Omp31 & BAB1_1639 & OmpA-like transmembrane domain & Outer membrane & 17014873 \\
12 & SodC & BAB2_0535 & Cu/Zn superoxide dismutase & Periplasm & 15039330 \\
13 & SurA & BAB1_0706 & Peptidyl-prolyl cis-trans isomerase & Periplasm & 17686554 \\
14 & Tig & BMEl1069 & Trigger factor & Cytoplasm & 17239499 \\
\hline
\end{tabular}

Abbreviations: TMH, transmembrane helixes; Adhesin Prob., Adhesin probability; Con-ed represents the conserved proteins among five other genomes from virulent B. abortus strain 9-941, B. melitensis strains $16 \mathrm{M}$ and ATCC 23457, and B. suis strains 1330 and ATCC 23445; Host Simil., similarity to human and mouse genomes.

proteins, four proteins are outer membrane proteins. The other nine proteins are located in cytoplasm (5 proteins), periplasm (4 proteins), and cytoplasmic membrane (1 protein).

For vaccine development against Brucella infections where $\mathrm{T}$ cell response is critical, subcellular localization is not usually an issue since a $\mathrm{T}$ cell response could be directed to any protein target. Our curated results confirm that protective Brucella antigens may occur in different subcellular locations.

\section{Prediction of potential Brucella vaccine targets}

Reverse vaccinology is an emerging vaccine development approach that starts with the prediction of vaccine targets using bioinformatics screening of an entire genome of a pathogenic organism [17]. As part of VIOLIN, Vaxign is the first web-based vaccine design program that predicts vaccine targets based on reverse vaccinology $[18,19]$. The Vaxign computational pipeline includes the following features: subcellular localization, topology (transmembrane helices and beta barrel structure), adhesin probability, similarity to other pathogen sequences, similarity to host genome sequences (e.g., human or mouse), and MHC class I and II epitope predictions. To predict Brucella vaccine targets, all 10 sequenced Brucella genomes available in NCBI RefSeq were used for a Vaxign analysis.

As with other intracellular pathogens, protection against Brucella infections requires cell-mediated immunity (CMI). Secreted pathogen proteins are likely to stimulate cytotoxic T lymphocyte (CTL) responses [20]. However, no Brucella protein has been found to be secreted in any in vitro culture in a standard culture medium. An O-sialoglycoprotein endopeptidase (Gcp; RefSeq: YP_415230.1) in B. abortus strain 2308 was identified by Vaxign to be a potential secreted protein.
This protein is also conserved in the other virulent $B$. abortus, B. melitensis, and B. suis strains.

Vaxign was used to predict Brucella outer membrane proteins (OMP) as potential vaccine targets using $B$. abortus strain 2308 genome [6] as the seed genome (Figure 3). Among 3034 proteins in this genome, 32 were identified as OMPs. These OMPs from B. abortus strain 2308 are listed in Table 3. Some specific groups such as cell wall/membrane/envelope biogenesis and cell motility were enriched based on the COG analysis [21]. Two proteins among the 32 OMPs contain more than one transmembrane spanning region each. These two proteins are excluded for further consideration since the presence of multiple transmembrane spanning regions may make the purification of such recombinant proteins difficult [22]. Adhesins present in microbial pathogens are essential for bacterial invasion and survival and represent possible targets for vaccine development. If only adhesins are considered, 10 out of the remaining 30 proteins have a probability $<0.51$ for being an adhesin and hence were discarded. Fifteen out the remaining 20 proteins are conserved in the genomes from virulent B. abortus strain 9-941, B. melitensis strain $16 \mathrm{M}$ and ATCC 23457, and B. suis strains 1330 and ATCC 23445. Each of these strains is pathogenic to humans. One protein (BAB1_1944) has homology with human and mouse proteomes. Among these 14 predicted Brucella vaccine targets, Omp25 (YP_414164.1) and Omp31-1 (YP_414995.1) have been verified to be protective Brucella antigens [23,24]. The list of predicted targets also includes two flagellar hook proteins FlgE (YP_419225.1) and FlgK (YP_419224.1), one porin protein Omp2b (YP_414102.1), and two TonB-dependent receptor proteins BAB1_1367 and BAB2_1150. The roles of these potential proteins as protective Brucella 


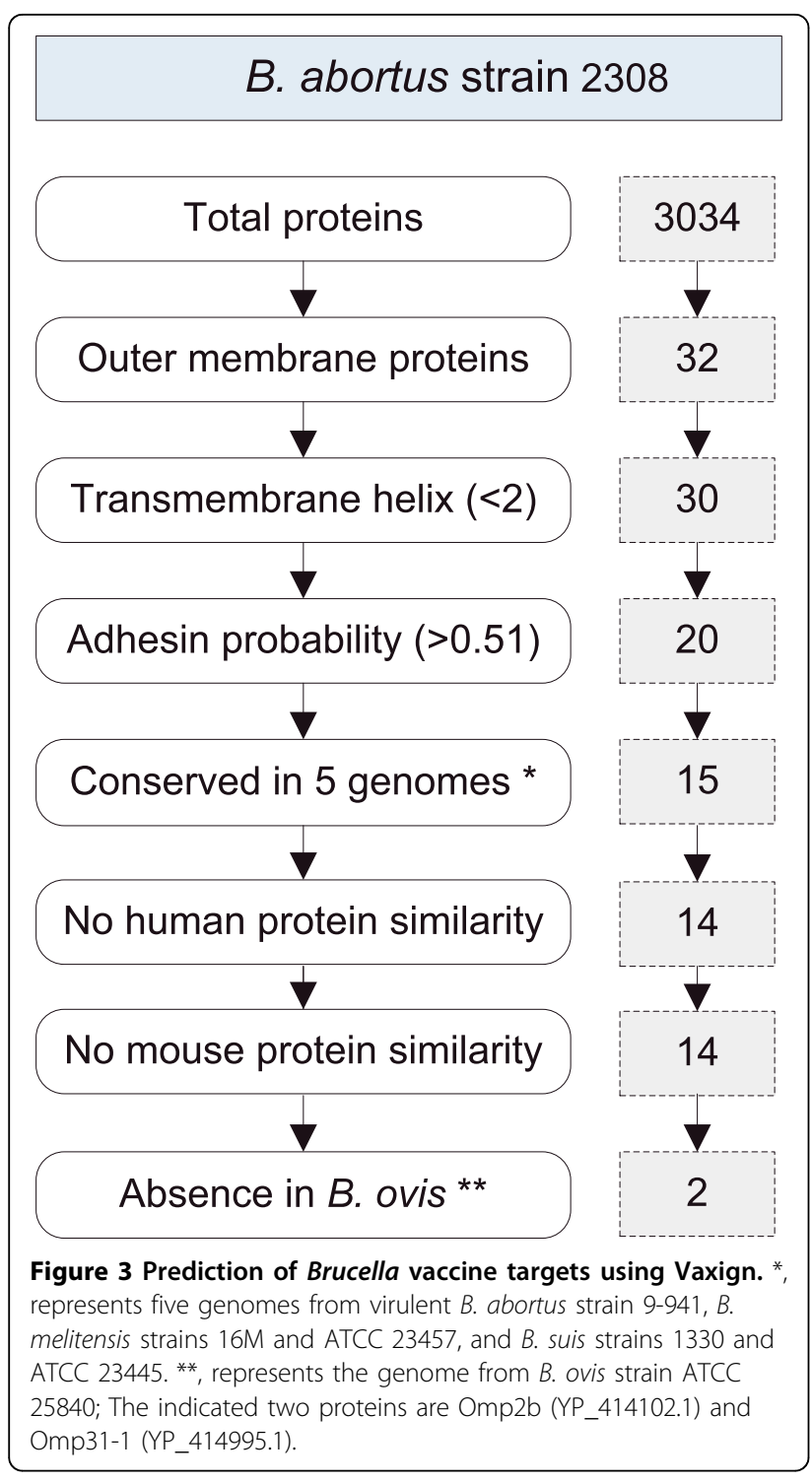

antigens have not been studied. The flagellar protein FlgJ appears in B. abortus strains 2308 and 9-941, B. melitensis strain 16M, and B. suis strain ATCC 23445; however, FlgJ is absent from $B$. suis strain 1330 and $B$. microti strain CCM 4915. Brucella flagellar genes have recently been found important in Brucella survival in vivo [25]. It remains unknown whether these Brucella flagellar genes can be used for Brucella vaccine development.

To develop a human Brucella vaccine, those Brucella proteins that exist in Brucella strains pathogenic to humans but are absent in Brucella strains that are nonpathogenic to humans would be ideal for vaccine development. Our studies have identified two proteins, Omp2b (YP_414102.1) and Omp31-1 (YP_414995.1), which are conserved in the above mentioned virulent $B$. abortus, $B$. melitensis, and B. suis strains that are pathogenic to humans, but absent from $B$. ovis that is nonpathogenic to humans. Omp2b and Omp31 are two major outer membrane proteins in $B$. abortus [26]. It is likely that these two proteins are critical for human-specific Brucella infections. If a human Brucella vaccine is developed, these two proteins are considered as priority antigens. A further bioinformatics analysis indicates that the porin protein Omp2b does not exist in live attenuated $B$. abortus vaccine strain 19, suggesting that Omp2b likely contributes to the attenuation of this mutant. Omp2b also exists in B. canis that is weakly pathogenic to humans. However, Omp31-1 does not exist in B. canis.

Vaxign identified 46 Brucella periplasmic proteins that are conserved in all B. abortus, B. melitensis, and B. suis genomes and lack sequence similarity with proteins in human or mouse genomes. The values of these proteins for vaccine development also deserve further analysis. Using the same criteria (sequence conservation and dissimilarity from human or mouse proteins), Vaxign detected approximately 1,000 cytoplasmic proteins. It is impractical to individually test this high number of proteins for vaccine development. Considering only five cytoplasmic proteins have been experimentally confirmed to be protective antigens out of 1,000 conserved cytoplasmic proteins (Table 2), it is much less likely that cytoplasmic proteins serve as protective antigens compared to outer membrane and periplasmic proteins.

Vaxign also contains an epitope prediction component that can predict MHC class I and II binding epitopes [19]. The addition of epitope prediction allows further analysis for the existence of potential Brucella vaccine targets.

\section{Other programs in VIOLIN}

VIOLIN provides user-friendly web interface for users to query Brucella vaccine data in VIOLIN. For example, Vaxquery is a user-friendly web query tool to query vaccine data (Figure 4).

VIOLIN VBLAST is a customized BLAST sequence similarity search program. The BLAST library in VBLAST includes those vaccine-associated genes, including protective antigens, virulent factors whose mutations lead to live attenuated vaccine development, and host protective immune factors. These vaccine-associated genes can also be queried through our Vaxgen web interface.

Two VIOLIN programs Vaxjo and Vaxvec permit analysis of vaccine adjuvants and vaccine vectors. The adjuvants used for Brucella vaccine development include Complete and Incomplete Freund's Adjuvants, CpG, Cholera toxin (CT) adjuvant, Maltose binding protein (MBP). 
Table 3 Vaxign-predicted vaccine targets from B. abortus strain 2308

\begin{tabular}{|c|c|c|c|c|c|c|c|c|}
\hline$\#$ & Locus Tag & RefSeq \# & Symbol & TMH & $\begin{array}{l}\text { Adhesin } \\
\text { Prob. }\end{array}$ & $\begin{array}{l}\text { Con- } \\
\text { ed }\end{array}$ & $\begin{array}{l}\text { Host } \\
\text { Simil. }\end{array}$ & Protein Notes \\
\hline \multicolumn{9}{|c|}{ Cell Motility } \\
\hline 1 & BAB2_1097 & YP_419224.1 & FlgK & 0 & 0.535 & $x$ & & flagellar hook-associated protein FlgK \\
\hline 2 & BAB2_1098 & YP_419225.1 & FlgE & 0 & 0.749 & $x$ & & flagellar hook protein FlgE \\
\hline 3 & BAB1_0260 & YP_413736.2 & Flg」 & 0 & 0.656 & & & $\begin{array}{l}\text { Flagellar protein FlgJ:Mannosyl-glycoprotein endo-beta-N- } \\
\text { acetylglucosamidase }\end{array}$ \\
\hline 4 & BAB1_1726 & YP_415076.1 & & 1 & 0.229 & $x$ & & hypothetical protein \\
\hline \multicolumn{9}{|c|}{ TonB-dependent Receptor Protein: Inorganic Ion Transport and Metabolism } \\
\hline 5 & BAB2_0233 & YP_418452.1 & & 0 & 0.405 & $x$ & & TonB-dependent receptor protein \\
\hline 6 & BAB2_1150 & YP_419272.1 & & 0 & 0.691 & $x$ & & $\begin{array}{l}\text { TonB-dependent receptor protein:Pollen allergen Poa pIX/Phl pVI, } \\
\text { C-terminal }\end{array}$ \\
\hline 7 & BAB1_1367 & YP_414742.1 & & 0 & 0.655 & $x$ & & TonB-dependent receptor protein \\
\hline \multicolumn{9}{|c|}{ ATP/GTP-binding Site Motif A (P-loop): Porin, Alpha proteobacteria type } \\
\hline 8 & BAB1_0659 & YP_414101.1 & Omp2a & 0 & 0.611 & & & Porin, alpha proteobacteria type \\
\hline 9 & BAB1_0660 & YP_414102.1 & Omp2b & 0 & 0.585 & $x$ & & Porin, alpha proteobacteria type \\
\hline \multicolumn{9}{|c|}{ Cell wall/membrane/envelope Biogenesis } \\
\hline 10 & BAB1_0045 & YP_413545.1 & & 0 & 0.388 & $x$ & & Bacterial surface antigen (D15) \\
\hline 11 & BAB1_0115 & YP_413611.1 & & 0 & 0.793 & $x$ & & outer membrane protein, putative \\
\hline 12 & BAB1_0116 & YP_413612.1 & & 0 & 0.58 & $x$ & & outer membrane protein, putative \\
\hline 13 & BAB1_0707 & YP_414149.1 & & 0 & 0.635 & $x$ & & Organic solvent tolerance protein \\
\hline 14 & BAB1_0722 & YP_414164.1 & Omp25 & 0 & 0.554 & $x$ & & OmpA-like transmembrane domain \\
\hline 15 & BAB1_1176 & YP_414567.1 & & 1 & 0.408 & $x$ & & Bacterial surface antigen (D15) \\
\hline 16 & BAB1_1226 & YP_414612.1 & & 3 & 0.571 & $x$ & & MotY protein: OmpA/MotB domain \\
\hline 17 & BAB1_1302 & YP_414685.1 & RopB & 1 & 0.815 & $x$ & & hypothetical protein \\
\hline 18 & BAB1_1579 & YP_414943.1 & & 1 & 0.669 & $x$ & & OmpW family \\
\hline 19 & BAB1_1639 & YP_414995.1 & $\begin{array}{l}\text { Omp31- } \\
1\end{array}$ & 0 & 0.736 & $x$ & & OmpA-like transmembrane domain \\
\hline 20 & BAB1_1707 & YP_415057.1 & & 0 & 0.371 & $x$ & & MotY protein: OmpA/MotB domain \\
\hline 21 & BAB2_0314 & YP_418525.1 & & 1 & 0.649 & $x$ & & heat resistant agglutinin 1 precursor \\
\hline 22 & BAB1_0963 & YP_414386.1 & & 0 & 0.415 & $x$ & & Outer membrane efflux protein \\
\hline \multicolumn{9}{|c|}{ Replication, Recombination and Repair } \\
\hline 23 & BAB2_0636 & YP_418811.1 & & 0 & 0.299 & $x$ & & DNA topoisomerase I \\
\hline 24 & BAB1_0121 & YP_413617.1 & & 0 & 0.162 & $x$ & $x$ & DEAD/DEAH box helicase \\
\hline \multicolumn{9}{|c|}{ Lipid Transport and Metabolism } \\
\hline 25 & BAB1_0967 & YP_414390.1 & & 0 & 0.764 & & & Membrane protein involved in aromatic hydrocarbon degradation \\
\hline \multicolumn{9}{|c|}{ Posttranslational Modification, Protein Turnover, Chaperones } \\
\hline 26 & BAB1_1944 & YP_415281.1 & & 1 & 0.518 & $x$ & & PpiC-type peptidyl-prolyl cis-trans isomerase \\
\hline \multicolumn{9}{|c|}{ Unknown Function } \\
\hline 27 & BAB1_1705 & YP_415055.1 & & 0 & 0.594 & $x$ & & TPR repeat:Molluscan rhodopsin C-terminal tail \\
\hline 28 & BAB1_1854 & YP_415198.1 & & 0 & 0.759 & & & hypothetical protein \\
\hline 29 & BAB2_0071 & YP_418316.1 & & 0 & 0.492 & $x$ & & hypothetical protein \\
\hline 30 & BAB1_0069 & YP_413569.1 & & 0 & 0.886 & & & hypothetical protein BAB1_0069 \\
\hline 31 & BAB1_0897 & YP_414322.1 & & 2 & 0.279 & $x$ & & Antifreeze protein, type I \\
\hline 32 & BAB1_0942 & YP_414367.1 & & 1 & 0.346 & $x$ & & RNA-binding region RNP-1 (RNA recognition motif) \\
\hline
\end{tabular}




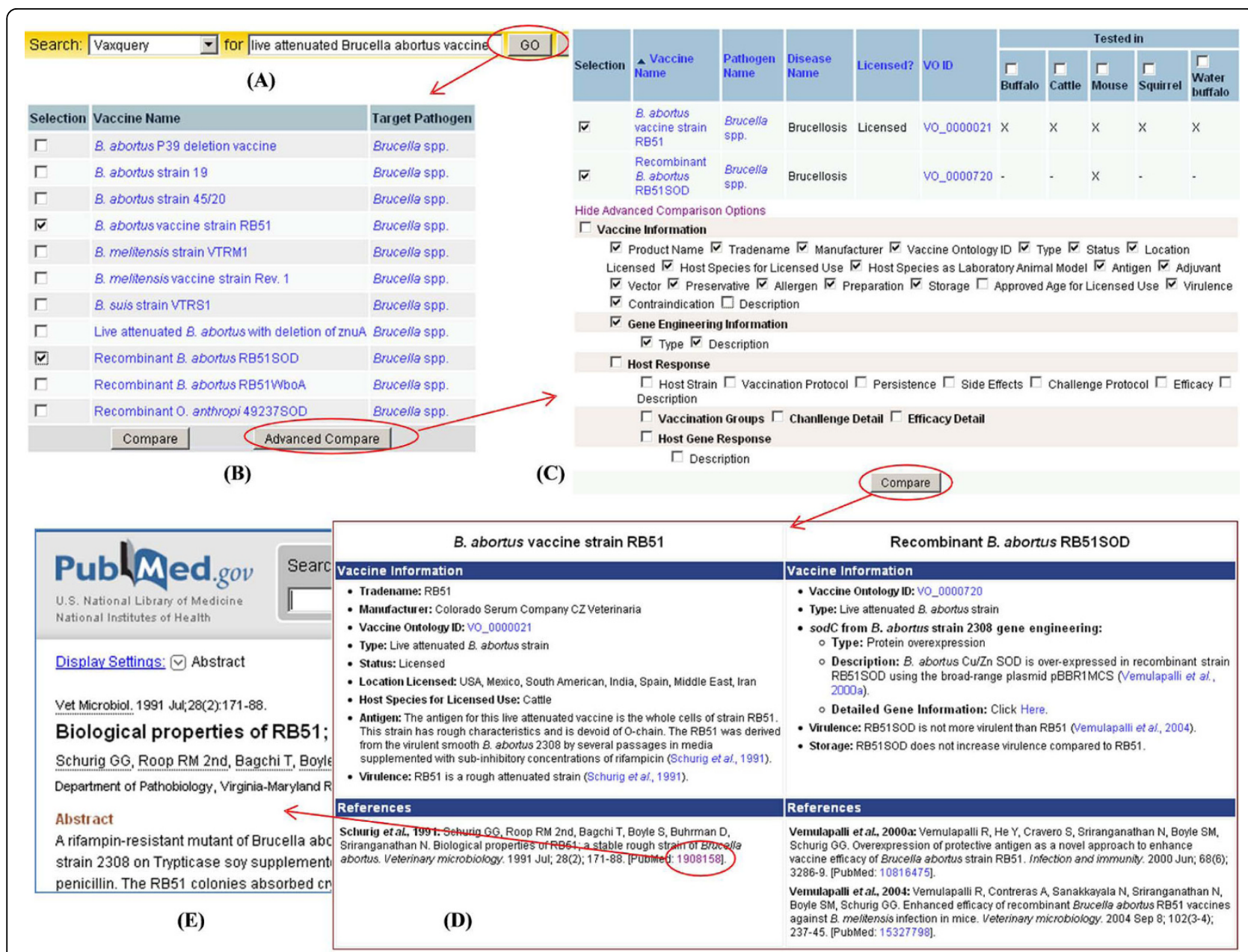

Figure 4 Brucella vaccine analyses using VIOLIN Vaxquery. After typing the "live attenuated Brucella abortus vaccines" in the Vaxquery query bar (A), 11 Brucella vaccines were displayed (B). Two vaccines (RB51 and RB51SOD) were chosen for advanced comparison (B). After query settings were chosen (C), the results were displayed (E). All curated information has associated references which can be linked to PubMed (E).

Additionally, VIOLIN contains the information for host responses to Brucella vaccines. Animal response information can be searched through VIOLIN Vaxar (http://www.violinet.org/vaxar). Currently, annotated information for 68 host response studies of Brucella vaccines is available in Vaxar. VIOLIN contains many pages that are associated with other vaccine related topics, such as vaccine conferences, manufacturers, and useful web links.

\section{Discussion}

A large number of vaccine-related databases exist on the web. There are many government-supported vaccine databases. For example, the Centers for Disease Control and Prevention (CDC) maintain a Vaccine Information Statements (VISs) system (http://www.cdc.gov/vaccines/ pubs/vis/default.htm). The Center for Biologics Evaluation and Research (CBER) under the Food and Drug Administration (FDA) regulates vaccine products and posts relevant information in their vaccine site: http:// www.fda.gov/cber/vaccines.htm. There is also a Vaccine Adverse Event Reporting System (VAERS, http://vaers. hhs.gov/), co-sponsored by FDA and CDC in USA. Many agent-specific databases are also available, for example, the HIV vaccine resource (http://www3.niaid. nih.gov/research/topics/HIV/vaccines/default.htm) created by the National Institute of Allergy and Infectious Diseases (NIAID) at the National Institutes of Health (NIH). Other vaccine resources include, the Vaccine Page: http://www.vaccines.org/), the Vaccine Resource Library (PATH, http://www.path.org/vaccineresources/), and the Immunization Action Coalition (http://www. immunize.org/). These databases primarily focus on available information concerning existing licensed vaccines and vaccine regulation. VIOLIN is unique in that it stores and analyzes research data concerning commercial vaccines and vaccines under clinical trial or in early stages of development [8]. 
The development of the Vaccine Ontology (VO) is a community effort and involves many experts in the vaccine and biomedical ontology communities [27]. With the large number of vaccine data types and publications available, $\mathrm{VO}$ is developed as an efficient strategy for vaccine data standardization, retrieval, and integration. VO makes it possible for computer programs to understand various vaccine types and research data associated with different vaccines. VO will also help to ensure that data is annotated in a way that ensures comparability. Therefore, VO-based software programs can be developed to support high throughput vaccine data processing and analysis. We are currently developing a VObased literature mining and curation program that would increase the efficacy of vaccine literature mining and manual curation. The VO-based literature mining program will also relieve the burden of continuous database updating. VO will also be used to integrate all vaccine data in VIOLIN, making vaccine information exchange more efficient.

Compared to the traditional vaccine development approach that starts from a wet laboratory, reverse vaccinology begins with dry laboratory bioinformatics analysis, which makes the vaccine development more specific and efficient. Reverse vaccinology was first used by Rino Rappuoli in the development of a vaccine against serogroup B Neisseria meningitidis (MenB), the major cause of sepsis and meningitis in children and young adults [28]. Since then, this strategy has been applied to many other pathogens such as Bacillus anthracis [29], Streptococcus pneumoniae [30], and Mycobacterium tuberculosis [31]. While the criteria for vaccine prediction are known and many individual programs are available, it is still time consuming and requires expertise in these individual programs to predict vaccine targets using genome sequences. Vaxign is the first web-based automated pipeline that identifies potential vaccine targets based on the reserve vaccinology strategy [19]. Vaxign has been applied successfully to predict vaccine targets for uropathogenic E. coli [19]. This study demonstrated that Vaxign can predict novel Brucella vaccine targets. Experimental verification of many of these targets is currently under way. Vaxign also contains a program to predict immune epitopes that bind to MHC class I and II molecules in different animal species. Studies analyzing and ranking potential immune epitopes from predicted Brucella proteins are in progress. Promising epitopes will be tested in a wet laboratory.

VIOLIN is also associated with other existing data resources. For example, many VIOLIN programs (e.g., Vaxign and Protegen) obtain Brucella genome sequences and share Brucella gene annotations with the web-based Pathogen-host Interaction Data Integration and Analysis System (PHIDIAS, http://www.phidias.us) [32]. PHIDIAS focuses on the analysis of pathogen-host interactions. Additionally, PHIDIAS contains the Brucella Bioinformatics Portal, a web-based portal with a special emphasis on Brucella genome annotation and literature mining [7]. PHIDIAS and BBP, also developed in our group, integrate more than 20 existing data resources. The close interaction between PHIDIAS/BBP and VIOLIN makes bioinformatics analysis of Brucella vaccines and vaccine targets more efficient.

VIOLIN currently includes vaccine data for 73 pathogens. The VIOLIN methods described for Brucella vaccine analysis in this report are generic and also feasible for vaccine studies for other pathogens. It is noted that Brucella is one of the most annotated pathogens among these 73 pathogens listed in VIOLIN. The vaccine information for many pathogens is not systematically annotated to the extent of Brucella vaccines. More work and collaborations with the research experts in these pathogens are necessary to curate and analyze vaccines and vaccine candidates for these pathogens.

\section{Conclusions}

VIOLIN provides manually curated Brucella vaccine data and ontology representation of these vaccines using the Vaccine Ontology (VO). Many tools are developed in VIOLIN to support literature mining and data curation. Examples of data stored in the VIOLIN database include protective Brucella antigens and host responses induced by different Brucella vaccines. Brucella vaccine targets may be predicted using the VIOLIN Vaxign program. Various Brucella vaccine data can be queried using user-friendly web query programs in VIOLIN. The VIOLIN approach is generic and can be used for analyses of vaccines against other pathogens and infection diseases.

\section{Methods}

Literature mining of Brucella vaccines using VIOLIN: The information of all PubMed papers associated with Brucella vaccine and vaccination were downloaded from the PubMed web service. The literature contents were processed using VIOLIN literature mining pipelines [8]. The processed results are available for users to analyzed using individual VIOLIN literature mining programs.

Bioinformatics curation of Brucella vaccines in VIOLIN: Brucella vaccine curation was performed on the VIOLIN web page using the Limix literature mining and curation system [7]. Limix allows data curators to submit data to the website, data reviewers to review and approve the submitted data, and eventual publication of high quality data. Specifically, a VIOLIN curator curates and compiles relevant information on vaccine 
information from peer-reviewed journals, books, and credible websites. The curated information is initially saved as a draft document and, when completed, is submitted to a MySQL database. The data submitted is initially invisible to the public and subject to critical review by an expert reviewer. Once approved, data becomes public and available for users to query. The database administrator manages users' accounts and curation tasks. The VIOLIN database is routinely maintained by the database administrator. Published database content is periodically reviewed to ensure that new, pertinent information is captured. When new information is found, a curator and/or a domain expert will update the database content using the standard procedure described above. In addition, the VIOLIN team also periodically emails the authors of new vaccine research publications and encourages them to submit their data through the VIOLIN online submission system. VIOLIN also includes internally developed scripts to automatically update gene annotations based on updated records from existing databases (e.g., NCBI Gene database).

VO representation of Brucella vaccines: Manually curated Brucella vaccines are entered into VO by following the VO development standards [27]. The VO is edited by Protégé (http://protege.stanford.edu/). The $\mathrm{FACT}++$ OWL reasoner [10] is used to obtain inferred Brucella vaccine hierarchy.

Vaxign prediction of Brucella vaccine targets: All ten Brucella genomes stored in the NCBI RefSeq database were used for prediction of Brucella vaccine targets. The genome of $B$. abortus strains 2308 was used as a seed genome. The other genomes include five sequenced virulent strains from three main pathogenic Brucella species: B. abortus strain 9-941), B. melitensis strains $16 \mathrm{M}$ and ATCC 23457, and B. suis strains 1330 and ATCC 23445. These strain are pathogenic to human. The genome of Brucella vaccine strain S19 was also included in this study for comparison purposes. The other three Brucella genomes are from B. ovis strain ATCC 25840, B. canis ATCC 23365, and B. microti strain CCM 4915. More Brucella genomes have been sequenced and available at http://www.broadinstitute.org/annotation/genome/brucella_group. Since the annotations are not yet finished and their records are not stored in the NCBI RefSeq database, these genomes were not typically used in this study. The Vaxign pipeline was executed by using the Brucella genomes as input data. The processed results were stored in the Vaxign database. The Vaxign web query interface was used to query and analyzed the predicted results.

Query of Brucella vaccine information in VIOLIN: All manually curated or computational processed data can be queried through various VIOLIN web pages.
Selected query functions are described in detail in the body of this manuscript.

List of Abbreviations

COG: The Clusters of Orthologous Groups; GO: Gene Ontology; Limix: Literature Mining and Curation System; MeSH: Medical Subject Headings; NLM: National Library of Medicine; NCBI: National Center for Biotechnology Information; NCBO: National Center for Biomedical Ontology; OBO: Open Biomedical Ontologies; OWL: Web Ontology Language; SOD: Superoxide Dismutase; VIOLIN: Vaccine Investigation and Online Information Network; VO: Vaccine Ontology; W3C: World Wide Web Consortium.

\section{Competing interests}

The authors declare that they have no competing interests.

\section{Authors' contributions}

$\mathrm{YH}$ : Brucella vaccine data analysis, VIOLIN design and project manager, manuscript writing.

ZX: Brucella vaccine data analysis, VIOLIN software developer and database administrator, manuscript editing.

\section{Acknowledgements}

This manuscript was supported by the NIH-NIAID grant R01Al081062. We appreciate Dr. George W. Jourdian's reviews and suggestions.

This article has been published as part of Immunome Research Volume 6 Supplement 1, 2010: Ninth International Conference on Bioinformatics (InCoB2010): Immunome Research. The full contents of the supplement are available online at http://www.immunome-research.com/supplements/6/S1.

\section{Published: 27 September 2010}

\section{References}

1. Corbel MJ: Brucellosis: an overview. Emerg Infect Dis 1997, 3(2):213-221.

2. Cloeckaert A, Verger JM, Grayon M, Paquet JY, Garin-Bastuji B, Foster G, Godfroid J: Classification of Brucella spp. isolated from marine mammals by DNA polymorphism at the omp2 locus. Microbes Infect 2001, 3(9):729-738.

3. Paulsen IT, Seshadri R, Nelson KE, Eisen JA, Heidelberg JF, Read TD, Dodson RJ, Umayam L, Brinkac LM, Beanan MJ, et al: The Brucella suis genome reveals fundamental similarities between animal and plant pathogens and symbionts. Proc Natl Acad Sci U S A 2002, 99(20):13148-13153.

4. Halling SM, Peterson-Burch BD, Bricker BJ, Zuerner RL, Qing Z, Li LL, Kapur V, Alt DP, Olsen SC: Completion of the genome sequence of Brucella abortus and comparison to the highly similar genomes of Brucella melitensis and Brucella suis. J Bacteriol 2005, 187(8):2715-2726.

5. DelVecchio VG, Kapatral V, Redkar RJ, Patra G, Mujer C, Los T, Ivanova N, Anderson I, Bhattacharyya A, Lykidis A, et al: The genome sequence of the facultative intracellular pathogen Brucella melitensis. Proc Natl Acad Sci U S A 2002, 99(1):443-448.

6. Chain PS, Comerci DJ, Tolmasky ME, Larimer FW, Malfatti SA, Vergez LM, Aguero F, Land ML, Ugalde RA, Garcia E: Whole-genome analyses of speciation events in pathogenic Brucellae. Infect Immun 2005, 73(12):8353-8361.

7. Xiang Z, Zheng W, He Y: BBP: Brucella genome annotation with literature mining and curation. BMC Bioinformatics 2006, 7(1):347.

8. Xiang Z, Todd T, Ku KP, Kovacic BL, Larson CB, Chen F, Hodges AP, Tian Y, Olenzek EA, Zhao $B$, et al: VIOLIN: vaccine investigation and online information network. Nucleic Acids Res 2008, 36(Database issue):D923-928.

9. Ashburner M, Ball CA, Blake JA, Botstein D, Butler H, Cherry JM, Davis AP, Dolinski K, Dwight SS, Eppig JT, et al: Gene ontology: tool for the unification of biology. The Gene Ontology Consortium. Nat Genet 2000, 25(1):25-29.

10. Tsarkov D, Horrocks I: FaCT++ Description Logic Reasoner: System Description. Proc of the Int Joint Conf on Automated Reasoning (IJCAR 2006), 4130 of Lecture Notes in Artificial Intelligence 2006, 292-297.

11. Xiang Z, He Y: Improvement of PubMed Literature Searching using Biomedical Ontology. The 1st International Conference on Biomedical 
Ontology (ICBO 2009) Nature Precedings Buffalo, NY, USA 2009: http:// precedingsnaturecom/documents/3491/version/1 August 24-26, 20092009

12. Hur J, Xiang Z, Feldman E, He Y: Ontology-based vaccine literature mining and indexing. Bio-Ontologies 2010: Semantic Applications in Life Sciences, 18th Annual International Conference on Intelligent Systems for Molecular Biology (ISMB): 2010; Boston, MA, USA. August 11-13 2010.

13. Ozgur A, Radev DR, Xiang Z, He Y: Mining of vaccine-associated IFN- $\gamma$ gene interaction networks using the Vaccine Ontology. Bio-Ontologies 2010: Semantic Applications in Life Sciences, 18th Annual International Conference on Intelligent Systems for Molecular Biology (ISMB): 2010; Boston, MA, USA. August 11-13. 2010

14. Brinkman RR, Courtot M, Derom D, Fostel J, He Y, Lord P, Malone J, Parkinson H, Peters B, Rocca-Serra P, et al: Modeling biomedical experimental processes with OBI. Journal of Biomedical Semantics 2010, 1(Suppl 1):S7.

15. He Y, Xiang Z, Todd T, Courtot M, Brinkman RR, Zheng J, Stoeckert CJ Jr., Malone J, Rocca-Serra P, Sansone SA, et al: Ontology representation and ANOVA analysis of vaccine protection investigation. Bio-Ontologies 2010: Semantic Applications in Life Sciences, 18th Annual International Conference on Intelligent Systems for Molecular Biology (ISMB): 2010; Boston, MA, USA. August 11-13. 2010

16. Ruttenberg A, Clark T, Bug W, Samwald M, Bodenreider O, Chen H, Doherty D, Forsberg K, Gao Y, Kashyap V: Advancing translational research with the Semantic Web. BMC Bioinformatics 2007, 8(Suppl 3):S2.

17. Rappuoli R: Reverse vaccinology. Curr Opin Microbiol 2000, 3(5):445-450.

18. Xiang $Z$, He Y: Vaxign: a web-based vaccine target design program for reverse vaccinology. Procedia in Vaccinology: 2009; 2nd Global Congress on Vaccines, December 7-9, 2008, Boston, MA, USA 2009, 23-29.

19. He $Y$, Xiang $Z$, Mobley HL: Vaxign: the first web-based vaccine design program for reverse vaccinology and applications for vaccine development. Journal of Biomedicine and Biotechnology 2010, 2010, (2010), Article ID 297505, 15 pages.

20. Kaufmann SH, Hess J: Impact of intracellular location of and antigen display by intracellular bacteria: implications for vaccine development. Immunol Lett 1999, 65(1-2):81-84.

21. Tatusov RL, Galperin MY, Natale DA, Koonin EV: The COG database: a tool for genome-scale analysis of protein functions and evolution. Nucleic Acids Res 2000, 28(1):33-36.

22. Serruto D, Rappuoli R, Pizza M: Meningococcus B: from Genome to Vaccine. Genomics, Proteomics and Vaccines John Wiley \& Sons Ltd.Grandi G 2004, 185-201.

23. Commander NJ, Spencer SA, Wren BW, MacMillan AP: The identification of two protective DNA vaccines from a panel of five plasmid constructs encoding Brucella melitensis 16M genes. Vaccine 2007, 25(1):43-54.

24. Cassataro J, Velikovsky CA, Bruno L, Estein SM, de la Barrera S, Bowden R, Fossati CA, Giambartolomei GH: Improved immunogenicity of a vaccination regimen combining a DNA vaccine encoding Brucella melitensis outer membrane protein 31 (Omp31) and recombinant Omp31 boosting. Clin Vaccine Immunol 2007, 14(7):869-874.

25. Fretin D, Fauconnier A, Kohler S, Halling S, Leonard S, Nijskens C, Ferooz J, Lestrate P, Delrue RM, Danese I, et al: The sheathed flagellum of Brucella melitensis is involved in persistence in a murine model of infection. Cell Microbiol 2005, 7(5):687-698.

26. Connolly JP, Comerci D, Alefantis TG, Walz A, Quan M, Chafin R, Grewal P, Mujer CV, Ugalde RA, DelVecchio VG: Proteomic analysis of Brucella abortus cell envelope and identification of immunogenic candidate proteins for vaccine development. Proteomics 2006, 6(13):3767-3780.

27. He $Y$, Cowell L, Diehl AD, Mobley HL, Peters B, Ruttenberg A, Scheuermann RH, Brinkman RR, Courtot M, Mungall C, et al: VO: Vaccine Ontology. The 1st International Conference on Biomedical Ontology (ICBO 2009) Nature Precedings Buffalo, NY, USA 2009, http://precedings.nature. com/documents/3553/version/1.

28. Pizza M, Scarlato V, Masignani V, Giuliani MM, Arico B, Comanducci M, Jennings GT, Baldi L, Bartolini E, Capecchi B, et al: Identification of vaccine candidates against serogroup $B$ meningococcus by whole-genome sequencing. Science 2000, 287(5459):1816-1820.

29. Ariel N, Zvi A, Grosfeld H, Gat O, Inbar Y, Velan B, Cohen S, Shafferman A: Search for potential vaccine candidate open reading frames in the Bacillus anthracis virulence plasmid pXO1: in silico and in vitro screening. Infect Immun 2002, 70(12):6817-6827.
30. Wizemann TM, Heinrichs JH, Adamou JE, Erwin AL, Kunsch C, Choi GH, Barash SC, Rosen CA, Masure HR, Tuomanen E, et al: Use of a whole genome approach to identify vaccine molecules affording protection against Streptococcus pneumoniae infection. Infect Immun 2001, 69(3):1593-1598

31. Betts JC: Transcriptomics and proteomics: tools for the identification of novel drug targets and vaccine candidates for tuberculosis. IUBMB Life 2002, 53(45):239-242.

32. Xiang Z, Tian Y, He Y: PHIDIAS: a pathogen-host interaction data integration and analysis system. Genome Biol 2007, 8(7):R150.

doi:10.1186/1745-7580-6-S1-S5

Cite this article as: $\mathrm{He}$ and Xiang: Bioinformatics analysis of Brucella vaccines and vaccine targets using VIOLIN. Immunome Research 2010 6(Suppl 1):S5

\section{Submit your next manuscript to BioMed Central and take full advantage of:}

- Convenient online submission

- Thorough peer review

- No space constraints or color figure charges

- Immediate publication on acceptance

- Inclusion in PubMed, CAS, Scopus and Google Scholar

- Research which is freely available for redistribution

Submit your manuscript at www.biomedcentral.com/submit
C) Biomed Central 